\title{
Metallic behavior in low-dimensional honeycomb SiB crystals: A first-principles prediction of atomic structure and electronic properties
}

\author{
Anders Hansson, ${ }^{*}$ F. de Brito Mota, ${ }^{\dagger}$ and Roberto Rivelino ${ }^{\ddagger}$ \\ Instituto de Física, Universidade Federal da Bahia, 40210-340 Salvador, Bahia, Brazil \\ (Received 8 May 2012; revised manuscript received 5 September 2012; published 14 November 2012)
}

\begin{abstract}
We present a detailed analysis of the atomic and electronic structure of a two-dimensional monolayer of boron and silicon elements within periodic density functional theory. The proposed h-SiB sheet is a structural analog of hexagonal boron nitride (h-BN) and exhibits a good structural stability, compared to the structure of silicene. The calculated cohesive energy of an infinite sheet of $\mathrm{h}-\mathrm{SiB}$ is of $4.71 \mathrm{eV} /$ atom, whereas the corresponding value for silicene is $4.09 \mathrm{eV} /$ atom. However, $\mathrm{h}-\mathrm{SiB}$ sheets are not able to be stacked into a three-dimensional graphitelike structure, leading to a new hexagonal phase. On the other hand, $\mathrm{h}-\mathrm{SiB}$ is predicted to roll up into single-walled silicon boron nanotubes (SWSiBNTs) of which we examine the electronic properties of some zigzag and armchair tubes. The strain energy of the SWSiBNTs are four to five times lower than the strain energy of the corresponding carbon nanotubes. In contrast to more polar honeycomb monolayers, the h-SiB sheet is not semiconducting or semimetallic. It has a delocalized charge density like graphene, but the $\pi$ band and the two highest occupied $\sigma$ bands are only partly filled. This results in a high density of states around the Fermi level and a metallic behavior of the $\mathrm{h}-\mathrm{SiB}$ sheet. Interestingly, all the low-dimensional h-SiB-based structures, including the smallest to the largest stable tubes studied here, are predicted to form metallic systems.
\end{abstract}

DOI: 10.1103/PhysRevB.86.195416

PACS number(s): 31.15.E-, 73.22.-f, 61.46.Fg, 61.48.De

\section{INTRODUCTION}

One of the most striking features that determine the properties of a material is its extent in space. A chemical structure arranged in quasizero, one, two, or three dimensions can display the most diverse properties already observed in nature. Peierls ${ }^{1}$ and Landau ${ }^{2}$ demonstrated that one-dimensional (1D) and two-dimensional (2D) crystals could not exist, because of their large in-plane thermal fluctuations. Later their arguments were extended by Mermin ${ }^{3}$ and were supported by a great deal of experiments. ${ }^{4}$ Anderson further demonstrated that electrons in low-dimensional systems always will experience strong (Anderson) localization ${ }^{5}$ due to impurities or defects, if their randomness is sufficiently large.

Nevertheless, the atomic monolayer of graphitegraphene-has been thoroughly studied theoretically since the 1940s. ${ }^{6-9}$ Until 2004 graphene was considered as an academic material, though useful as a theoretical toy model for understanding and describing other carbon-based materials. It was therefore a great surprise when Geim and Novoselov ${ }^{10,11}$ finally managed to isolate and identify graphene, which appeared to be stable in an ambient environment.

Since then, 2D structures have gained renewed interest and more monolayers have been reported, ${ }^{12-14}$ with special attention paid to silicene. ${ }^{15-22}$ For example hexagonal boron nitride ${ }^{23}(\mathrm{~h}-\mathrm{BN})$, the structural analog of graphene, has also been fabricated and found to be thermally and chemically stable under ambient conditions, as well as $\mathrm{MoS}_{2}, \mathrm{NbS}_{2}$, and $\mathrm{Bi}_{2} \mathrm{Sr}_{2} \mathrm{CaCu}_{2} \mathrm{O}_{x} \cdot{ }^{24}$ Recently, the artificial growth of one-atom-thick materials inside or on top of stable crystals has been experimentally possible. ${ }^{24}$ Various elemental honeycomb structures of group IV and binary compounds of groups III and V have been proposed and theoretically studied. ${ }^{21,25}$ Except for graphene, only silicon and germanium were predicted to be semimetallic as hon- eycomb monolayers, while the other binary compounds all are semiconductors. ${ }^{26-31}$ Reported honeycomb-structured thin films/multilayers are also semiconducting, although there are cases of 2D metallic surface states in (wurtzite) thin films of $\mathrm{ZnO}{ }^{32,33}$ However, this new world of $2 \mathrm{D}$ crystals is still greatly unexplored.

In another context, the group-III element boron, the lightest element able to form covalent bonds, has since the discovery of superconducting quaternary borocarbides ${ }^{34}$ and the high-temperature nonoxide superconductor $\mathrm{MgB}_{2}$ (Ref. 35), emerged as an interesting dopant. $\mathrm{MgB}_{2}$ consists of stacked boron honeycomb sheets, intercalated with $\mathrm{Mg}$ ions which stabilize the sheets. More recently boron has been reported as being involved in superconducting diamond, ${ }^{36}$ silicon carbide, ${ }^{37}$ and diamond cubic silicon, ${ }^{38}$ which all were heavily doped with boron. On the other hand, silicon boron compounds are still a poorly known group of boron-containing solids, for which various phases have been suggested. ${ }^{39-42}$

In the present study we propose a graphenelike structure made of silicon and boron, i.e., h-SiB in analogy with h-BN. By means of first-principles density functional calculations, we have determined geometry, discussed stability, and calculated electron structure, and moreover compared with isomorphic systems, particularly graphene, h-BN, and silicene. Different from graphene, which is a gapless semiconductor, and $\mathrm{h}$ $\mathrm{BN}$, which is known to be highly insulating, we predict the silicon boron sheets to form a metallic 2D material. The combination of the group-III element boron with the group-IV element silicon, in a honeycomb structure, will have overlap between the singly occupied $3 p_{z}$ orbital of silicon and the empty $2 p_{z}$ orbital of boron, which may form a partly filled conjugated system. Furthermore, we have demonstrated that $\mathrm{h}-\mathrm{SiB}$ forms metallic single-walled nanotubes, which are in some extent well comparable to carbon nanotubes. 


\section{COMPUTATIONAL DETAILS}

In the present calculations we utilize density functional theory (DFT) for periodic systems as implemented in the SIESTA code (version 3.0). ${ }^{43}$ The exchange and correlation functional is approximated with the Perdew-Burke-Ernzerhof (PBE) derivation ${ }^{44}$ of the generalized gradient approximation (GGA), which takes into account the semilocal exchange correlations which have significant impact on low-dimensional systems such as boron nitride and graphene nanoribbons. ${ }^{45}$ The interaction of the valence electrons with the ion cores is represented by the norm-conserving Troullier-Martins pseudopotentials. ${ }^{46}$ The basis set is the SIESTA default double- $\zeta$ with polarization for all atoms with cutoff radii defined by the energy shift of $0.1 \mathrm{eV}$, and the plane-wave cutoff determining the real-space grid is $200 \mathrm{Ry}$.

The Brillouin zone is sampled with the Monkhorst-Pack algorithm employing the grids $81 \times 81 \times 1$ and $1 \times 1 \times 81$ for sheets and tubes, $41 \times 41 \times 41$ for the silicon and diamond (primitive unit cells), and $31 \times 31 \times 11$ and $21 \times 21 \times 21$ for graphite and $\alpha$-rhombohedral boron $\left(\alpha-\mathrm{B}_{12}\right)$, respectively. For electronic relaxation the standard diagonalization is used with a density-matrix tolerance of $1.0 \times 10^{-4}$ as a convergence criterion. The structural relaxation considers both atomic coordinate and lattice parameter optimization with the conjugate gradient (CG) algorithm in vacuum. A force tolerance of $10 \mu \mathrm{eV} / \AA$ and a stress tolerance of $10 \mu \mathrm{eV} / \AA^{3}$ are the convergence criteria for the structural relaxation.

The structural stability is primarily evaluated with the cohesive energies $\left(E_{\text {coh }}\right)$ calculated according to Ashcroft and Mermin $^{47}$ [given by Eq. (1)]:

$$
E_{\mathrm{coh}}=E_{\mathrm{tot}}[X]+E_{\mathrm{tot}}[Y]-E_{\mathrm{tot}}[X Y] .
$$

$E_{\text {tot }}[X]$ and $E_{\text {tot }}[Y]$ are the total energies of the free atoms, here approximated by an 80 - $\AA$-wide simple cubic unit cell, and $E_{\mathrm{tot}}[X Y]$ is the total energy of the compound unit cell.

A tentative calculation of formation energies $\left(E_{\mathrm{form}}\right)$ as defined in Eq. (2) is also performed in analogy with Ref. 48 by using $\alpha-\mathrm{B}_{12}$, graphite, $\mathrm{N}_{2}$ gas, and silicon (diamond cubic) as standard states:

$$
E_{\mathrm{form}}=\frac{E_{\mathrm{tot}}[X Y]-n_{X} \mu_{X}-n_{Y} \mu_{Y}}{n_{X}+n_{Y}},
$$

where $n_{X}$ and $n_{Y}$ are the number of $X$ and $Y$ atoms per unit cell, and $\mu_{X}$ and $\mu_{Y}$ are the chemical potentials of atoms $X$ and $Y$ in the standard states.

Moreover, the energy associated with rolling up the sheets into tubes, i.e., the strain energy, which measures the stabilizing tension in hollow tubule structures are obtained from Eq. (3), as defined in Ref. 49:

$$
E_{\text {strain }}=E_{\text {coh }}[\text { sheet }]-E_{\text {coh }}[\text { tube }] .
$$

In the case of the SWSiBNTs, we have also performed $a b$ initio molecular dynamics (AIMD) simulations in the Nosé-Parrinello-Rahman NPT ensemble. The time step of these AIMD simulations is $1.0 \mathrm{fs}$ and the temperature is controlled with the Nosé thermostat at $500 \mathrm{~K}$ with a Nosé mass of $100.0 \mathrm{Ry} \mathrm{fs}^{2}$, while the pressure is controlled by the Parrinello-Rahman method at 0 Pa with a Parrinello-Rahman mass of $100.0 \mathrm{Ry}_{\mathrm{fs}}{ }^{2}$. The initial configurations are the
CG-optimized structures, with supercells of four tube unit cells and an intertube distance of $40 \AA$. To enable longer simulation times, the electronic structure calculations have been simplified in the following parts. For these cases, the DZ basis set, a mesh cutoff of $100 \mathrm{Ry}$ and a $1 \times 1 \times 15$ Monkhorst-Pack grid have been used. This is similar to the recent studies of melting single-walled silicon carbide nanotubes. ${ }^{50}$

\section{RESULTS AND DISCUSSION}

\section{A. Relaxed structures and stability}

The honeycomb structure of silicon and boron, h-SiB, has been relaxed according to the methods as described above, and it has been found to be planar with a bond length of $1.99 \AA$ (see Table I for comparison with other related systems). To evaluate our method and compare the calculated properties we also include similar structures, such as graphene, h-BN, silicene, and the corresponding standard states (graphite, nitrogen gas, diamond cubic silicon, and $\alpha-\mathrm{B}_{12}$ ), some single-walled carbon nanotubes, and diamond in the study. The notation $\alpha$-silicene is used for the planar sheet, while the most stable structure, which is buckled, is labeled $\beta$-silicene. ${ }^{22}$ Our calculated bond lengths of graphene and h-BN monolayer are $1.4 \%$ and $0.8 \%$ longer than the experimental values of graphite ${ }^{51}$ and bulk h-BN, ${ }^{52}$ respectively. In all monolayer calculations, the interplane distance is kept at $80 \AA$ by applying a stress constraint.

A general observation for the relaxed structures is that the lattice constants/bond lengths are slightly larger than the experimental values. A contributing factor, as expected, is the use of the GGA functional.

Since the $\mathrm{h}-\mathrm{SiB}$ is a structural analog of $\mathrm{h}-\mathrm{BN}$ and graphene, we also test the possibility of obtaining stable nanotubes. Moreover h-SiB exhibits an interesting structure such as those of $\mathrm{h}-\mathrm{BN}$ and $\mathrm{SiC}$, which also form nanotubes. The same notation for describing the geometry of the single-walled silicon boron nanotubes (SWSiBNTs) as for single-walled carbon nanotubes (SWCNTs) are used. Input geometries of the nanotubes were generated with the active server page application TubeASP using the lattice constant of the relaxed sheets.

All zigzag tubes up to $(10,0)$ and armchair tubes up to $(10,10)$ of both carbon and silicon boron have been relaxed. Due to the large number of atoms in the unit cells we accept a larger force tolerance of $1.0 \mathrm{meV} / \AA$. The SWSiBNTs $(5,5)$ $(7,7),(8,8)$, and $(9,9)$ are, however, only relaxed to the level $5.0 \mathrm{meV} / \AA$, because convergence to $1.0 \mathrm{meV} / \AA$ could not be reached after $2000 \mathrm{CG}$ steps. This is still considerably lower than the default force tolerance $40 \mathrm{meV} / \AA$ in SIESTA. Except for the smallest $(2,0)$ and $(3,0)$ zigzag SWSiBNTs, larger zigzag tubes have a perfect cylindrical shape (see Fig. 1). In the case of the armchair SWSiBNTs, only the $(3,3)$ tube does not relax to a hollow structure. The SWSiBNTs with a slow convergence have noncircular cross sections which are indications of lower strain energies (see Fig. 2), an effect that is observed for SWCNTs with larger radii.

In order to verify the structural stability of the SWSiBNTs, AIMD simulations were carried out with the largest armchair 
TABLE I. Calculated bond lengths, lattice constants, cohesive energies $\left(E_{\text {coh }}\right)$ and formation energies $\left(E_{\text {form }}\right)$ compared with available experimental data and other calculations. The calculated lattice angle of $\alpha-\mathrm{B}_{12}$ is $57.8^{\circ}$, while the corresponding experimental value is $58.04^{\circ}$. The $\alpha$-silicene ( $\beta$-silicene) structures refer to the flat (buckled) sheet, of which the $\alpha$-silicene is only relaxed in 2D.

\begin{tabular}{|c|c|c|c|c|}
\hline & Bond length $(\AA)$ & Lattice const. $(\AA)$ & $E_{\text {coh }}(\mathrm{eV} /$ atom $)$ & $E_{\text {form }}(\mathrm{eV} /$ atom $)$ \\
\hline Boron $\left(\alpha-\mathrm{B}_{12}\right)$ & & $\begin{array}{l}5.103 \\
5.051^{\mathrm{a}}\end{array}$ & 6.430 & \\
\hline (rhombic) & & & $5.80^{\mathrm{b}}$ & \\
\hline Graphite & & $\begin{array}{l}2.496,7.382 \\
2.4612,^{\mathrm{b}} 6.7079^{\mathrm{b}}\end{array}$ & $\begin{array}{l}7.863 \\
7.37^{\mathrm{b}}\end{array}$ & \\
\hline $\mathrm{N}_{2}$ (gas) & $\begin{array}{l}1.128 \\
1.0977^{\mathrm{b}}\end{array}$ & & $\begin{array}{l}4.771 \\
4.92^{b}\end{array}$ & \\
\hline Silicon & & $\begin{array}{l}5.522 \\
5.4305^{\mathrm{b}}\end{array}$ & $\begin{array}{l}4.790 \\
4.62^{\mathrm{b}}\end{array}$ & \\
\hline $\begin{array}{l}\text { h-BN (monolayer) } \\
\text { (bulk) }\end{array}$ & & $\begin{array}{l}2.524 \\
2.5040,{ }^{c} 6.6612^{c}\end{array}$ & 7.011 & -1.411 \\
\hline Graphene & & $\begin{array}{l}2.495 \\
2.469^{\mathrm{d}} \\
2.46^{\mathrm{e}}\end{array}$ & $\begin{array}{c}7.847 \\
7.936^{\mathrm{d}} \\
10.04^{\mathrm{e}}\end{array}$ & 0.016 \\
\hline Diamond & & $\begin{array}{l}3.594 \\
3.5670^{\mathrm{b}}\end{array}$ & 7.831 & 0.032 \\
\hline$\alpha$-silicene & 2.278 & $\begin{array}{l}3.945 \\
3.901^{\mathrm{f}}\end{array}$ & 4.058 & 0.732 \\
\hline$\beta$-silicene & $\begin{array}{l}2.311 \\
2.25^{\mathrm{e}}\end{array}$ & $\begin{array}{l}3.905 \\
3.83^{\mathrm{e}}\end{array}$ & $\begin{array}{l}4.091 \\
5.160^{\mathrm{e}}\end{array}$ & 0.699 \\
\hline h-SiB & 1.990 & 3.446 & 4.712 & 0.897 \\
\hline
\end{tabular}

${ }^{\mathrm{a}}$ Experimental (Ref. 53).

${ }^{\mathrm{b}}$ Experimental (Refs. 51 and 54).

${ }^{\mathrm{c}}$ Experimental (Ref. 56).

${ }^{\mathrm{d}}$ GGA calculated (Ref. 55).

${ }^{\mathrm{e}}$ LDA calculated (Ref. 25).

${ }^{\mathrm{f}}$ GGA calculated (Ref. 18).

and zigzag tubes and the $(5,5)$ tube, which relaxed to the least circular cross section.

Figure 3 displays the conservation of the cohesive energy during $1.5 \mathrm{ps}$ at $500 \mathrm{~K}$. The snapshots of the simulated
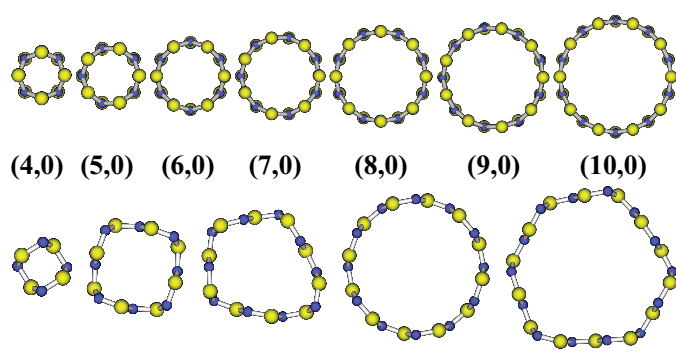

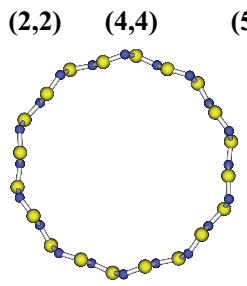

$(\mathbf{1 0 , 1 0 )}$
$(5,5)$

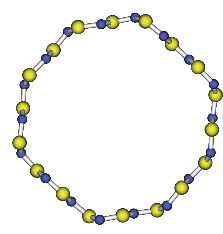

$(9,9)$
$(7,7)$

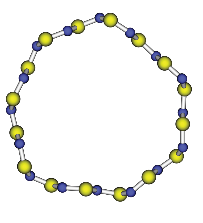

$(8,8)$
FIG. 1. (Color online) Cross sections of relaxed SWSiBNTs. Silicon and boron atoms are represented by yellow and blue spheres, respectively. structures are depicted in Fig. 4. Also, the thermal stability of the $(5,5)$ SWSiBNT was simulated up to $7 \mathrm{ps}$ with the same temperature and cohesive energy variations (see Fig. 5). These results give a good indication that these structures are stable at room temperature.

The ability to stack into 3D graphitelike structures was tested as well. Various initial configurations of stacked h-SiB

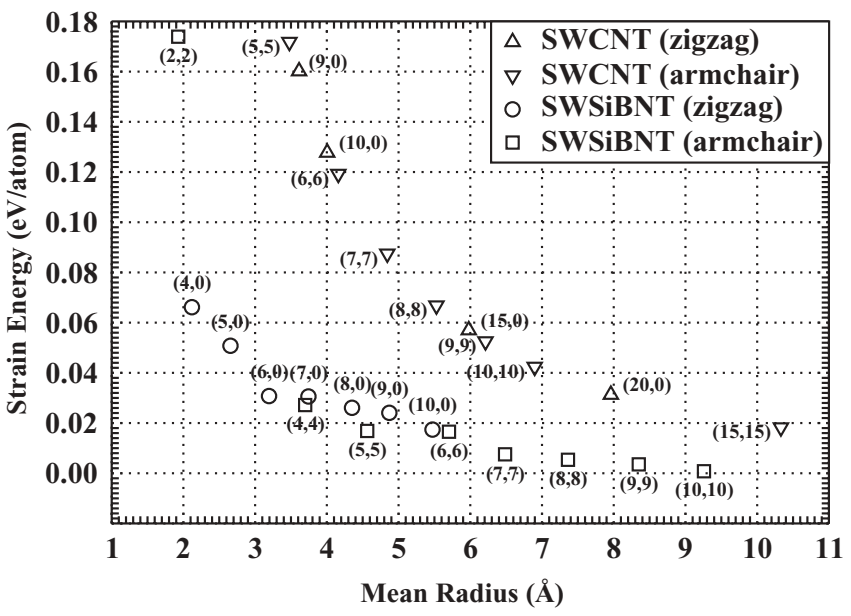

FIG. 2. Strain energies of relaxed SWCNTs and SWSiBNTs versus their mean radii. 


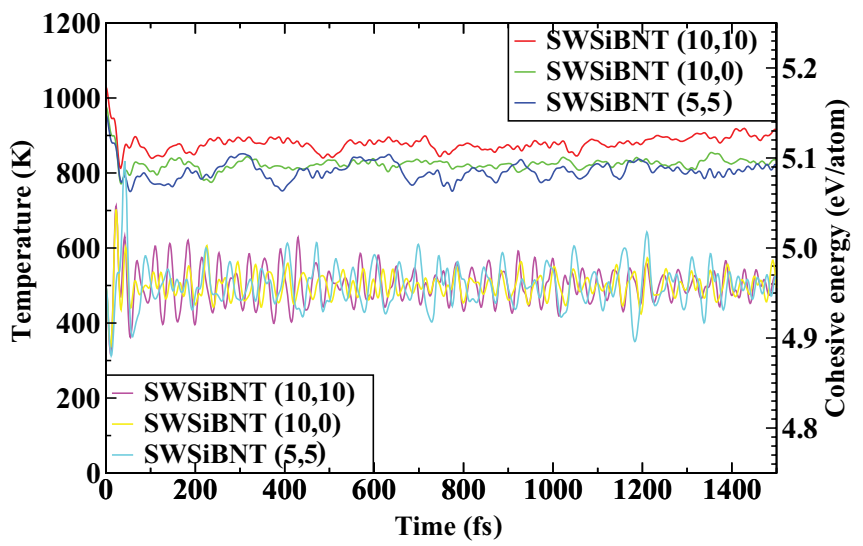

FIG. 3. (Color online) Temperature and cohesive energy versus time steps of the $(5,5),(10,0)$, and $(10,10)$ SWSiBNTs.

sheets have been relaxed. The resultant systems are best described as tilted wurtzitelike structures, in which covalent bonds between buckled hexagonal layers are formed by atoms of the same type. We believe that due to the reactivity between silicon and boron, the $\mathrm{h}-\mathrm{SiB}$ sheets are not able to form a hexagonal phase like graphite. However, we expect that one sheet can survive inside stable crystals such as $\alpha$-silicene inserted in graphitelike ultrathin stacks of aluminum nitride ${ }^{21}$ or on appropriate substrate. Recently silicene was successfully synthesized epitaxially on $\operatorname{Ag}(111){ }^{57}$

A fundamental quantity of crystal stability is the cohesive energy, which allows a direct comparison between experiments ${ }^{54}$ and first-principles calculations. These calculations [see Eq. (1)] require the total energy of the free atoms, which we obtain by using cubic unit cells with a lattice constant of $80 \AA$. The h-SiB sheet exhibits a good structural stability, with cohesive energy of $4.712 \mathrm{eV} /$ atom. This is, however, lower than the calculated cohesive energies for graphene with $7.86 \mathrm{eV} /$ atom and h-BN with $7.01 \mathrm{eV} /$ atom, only slightly lower than bulk silicon and $\mathrm{N}_{2}$ (with a difference of less than $0.10 \mathrm{eV} /$ atom), but larger than $\beta$-silicene with $4.09 \mathrm{eV} /$ atom. (a)

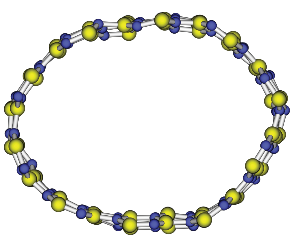

(b)

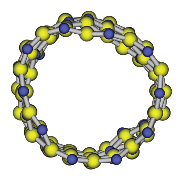

(c)

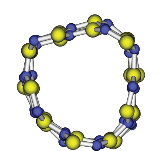

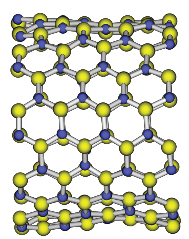
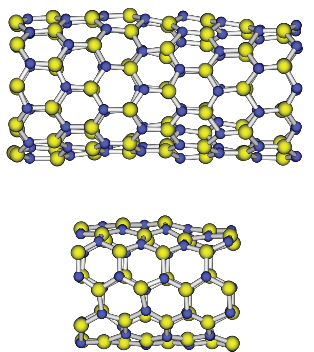

FIG. 4. (Color online) Snapshots of the SWSiBNTs after 1500 time steps (of $1 \mathrm{fs}$ ): (a) $(10,10)$, (b) $(10,0)$, and (c) $(5,5)$. Silicon and boron atoms are represented by yellow and blue spheres, respectively.

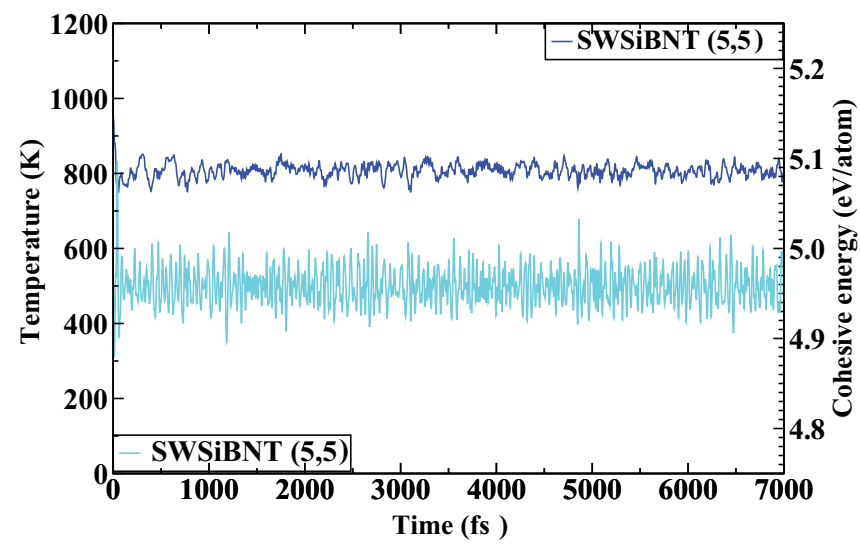

FIG. 5. (Color online) Temperature and cohesive energy versus time steps (of $1 \mathrm{fs}$ ) of the $(5,5)$ SWSiBNT.

For completeness, we further analyze the stability of the proposed $2 \mathrm{D} \mathrm{h}-\mathrm{SiB}$ system in terms of formation energy as defined in Ref. 48 and Eq. (2). As usual, the references are the standard states of the substances, i.e., $\alpha-B_{12}$, graphite, $N_{2}$, and cubic diamond silicon. The calculated formation energies per atom for most of the relaxed structures in this study are listed in Table I. From these values the $\beta$ structure of silicene is slightly more stable than its $\alpha$ structure, as expected for this system. $^{22}$

\section{B. Buckled sheets and single-walled nanotubes}

For several elementary and binary honeycomb structures a buckled phase is more stable than the planar phase. ${ }^{25}$ Generally the degree of buckling is described with the parameter $\Delta$ (see Fig. 6 for a definition of this parameter), which is the displacement/distance between planes of atom A and atom B in the primitive unit cell of the honeycomb lattice. One such structure is silicene, of which the planar $\alpha$-silicene is less stable than the buckled $\beta$-silicene, with a predicted $\Delta$ in the range of $0.44 \AA$ (Ref. 25) to $0.53 \AA$ (Ref. 58), and $0.51 \AA$ according to the present calculations.

To unveil whether the h-SiB sheets have buckled local minima, the variation in the total energy with respect to the buckling parameter $\Delta$ was determined from a series of fully
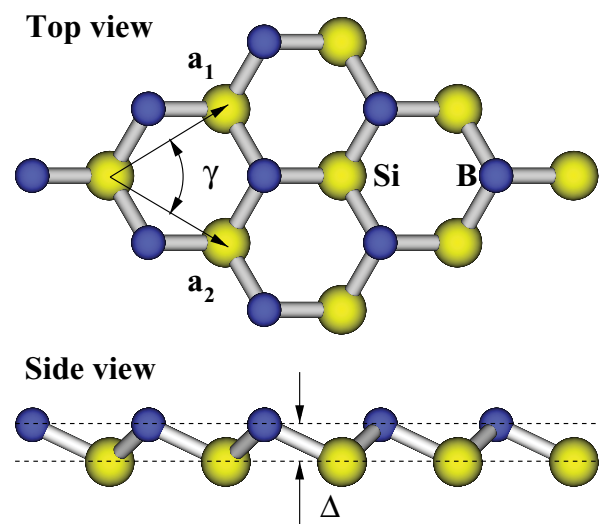

FIG. 6. (Color online) The buckling parameter $\Delta$ is defined as the distance between the planes of two atom types. $\gamma$ is the angle between the lattice vectors in the plane. 


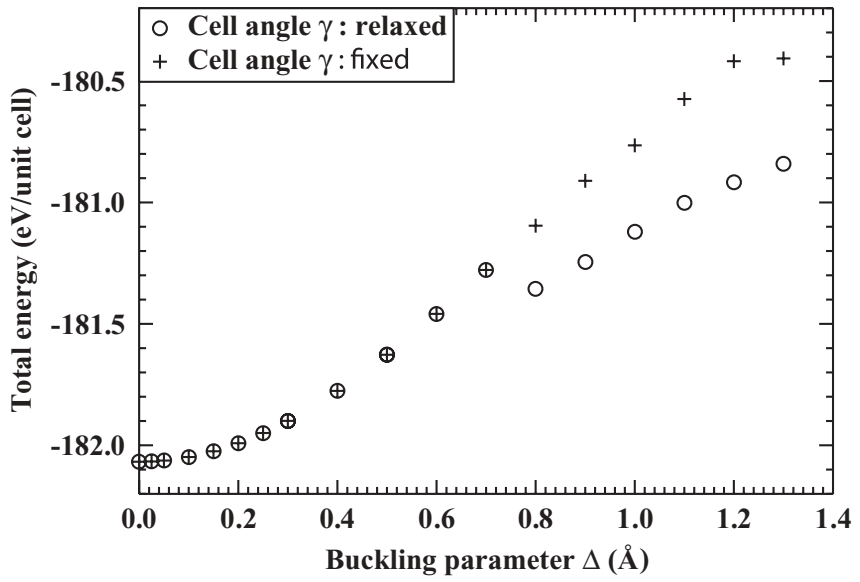

FIG. 7. Variation in the total energy of partly relaxed h-SiB with respect to the buckling parameter $\Delta$ (circles). The points denoted by + correspond to sheets relaxed with the additional constraint of a fixed angle $\gamma$.

relaxed buckled sheets, with $\Delta$ held fixed. The plotted total energy as a function of $\Delta$ (the circles in Fig. 7) clearly shows that the planar geometry is energetically more favorable than any buckled structures. The same series is repeated with the additional constraint of fixed cell angle $\gamma$ (the plus signs in Fig. 7). At the point where the total energy curves split (between 0.7 and $0.8 \AA$ ), the buckling is so high that covalent bonds begin to form between the silicon atoms which squeeze the honeycomb structure when the cell angle is relaxed.

Further tests of linearly independent displacements $(0.21$ and $0.02 \AA$ ) of a single atom in supercells of 2, 18, and 50 atoms, always give increased total energy. No larger scale ripples were observed in any of these relaxed supercells.

As discussed above, the strain energy associated with folding sheets into single-walled nanotubes ${ }^{49}$ also indicates the stability of the planar sheets. In this sense, we have therefore relaxed nanotubes with various radii of both graphene and $\mathrm{h}-\mathrm{SiB}$ sheets to examine the radial dependence and differences between these materials. The strain energies are calculated as defined in Eq. (3) and displayed in Fig. 2 with the strain energy/atom versus the mean radius for both types of nanotubes. The SWSiBNTs exhibit the same trend as the SWCNTs with a decreasing strain energy for increasing tube radius, but with about four to five times lower strain energy for a given radius. This strain energy is also well compared to the corresponding values of silicon nanotubes. ${ }^{59}$

\section{Electron densities and band structure of the low-dimensional $\mathrm{SiB}$ systems}

The h-SiB sheet has a charge density similar to that observed in graphene, exhibiting a kind of aromaticity as is already known for other silicon-based systems. ${ }^{60}$ Also h-SiB presents an overlapping between the $3 p_{z}$ orbital of $\mathrm{Si}$ and between the $2 p_{z}$ orbital of $\mathrm{B}$ (see Fig. 8), leading to an electronic behavior different from that observed for $\mathrm{h}-\mathrm{BN},{ }^{24}$ which has a charge density more localized in the nitrogen atoms. To guarantee a reliable description of the electron structure of our proposed h-SiB system, we have also studied its band structure and density of states (DOS) in comparison

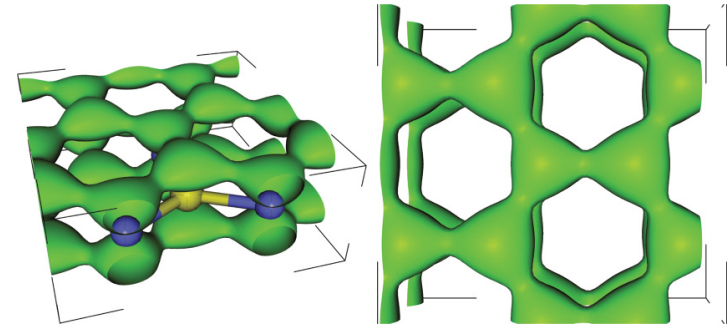

FIG. 8. (Color online) Probability density isosurfaces (at 50\% of maximum grid value) of the $\pi$ orbital at the $\Gamma$ point of the h-SiB sheet. Silicon and boron atoms are represented by yellow and blue spheres, respectively.

with these well-known and correlated systems, i.e., h-BN and graphene. Indeed, the calculated band structure and DOS, displayed in Fig. 9, demonstrate that h-BN has a direct band gap of $4.57 \mathrm{eV}$, graphene is a gapless semiconductor, while $\mathrm{h}-\mathrm{SiB}$ is predicted to form a metallic material. The band gap of h-BN was particularly studied by Arnaud et al. ${ }^{61}$ showing that the local density approximation (LDA), which (as GGA) systematically underestimates band gaps, gives a slightly smaller band gap of $4.46 \mathrm{eV}$ in comparison to experimental
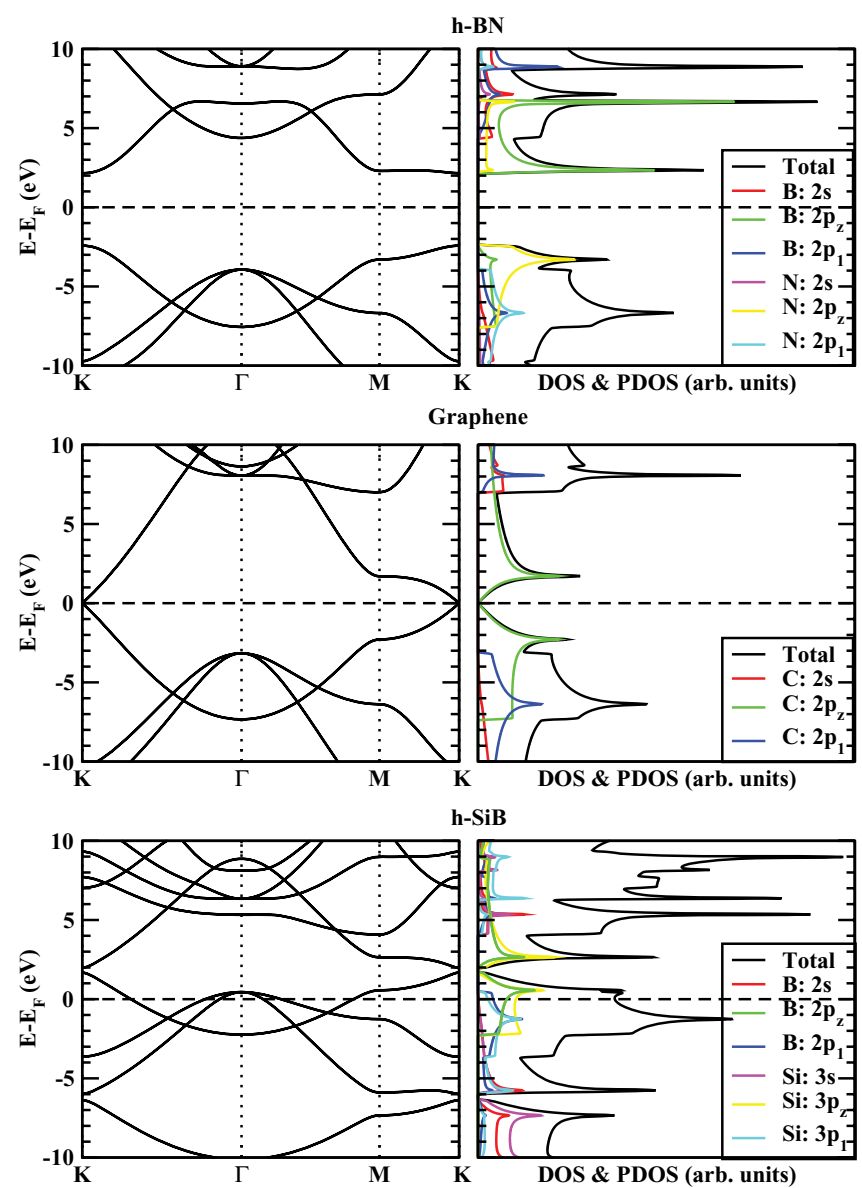

FIG. 9. (Color online) Band structures, DOS and PDOS of h-BN, graphene, and h-SiB. The Fermi energy is plotted with a dashed line. The PDOS of $n p_{-1}$ is equal to $n p_{1}$, therefore it is not shown in the figures. Also, the PDOS of the $3 d$ orbitals of silicon are excluded due to their minor contribution below and around the Fermi energy. 


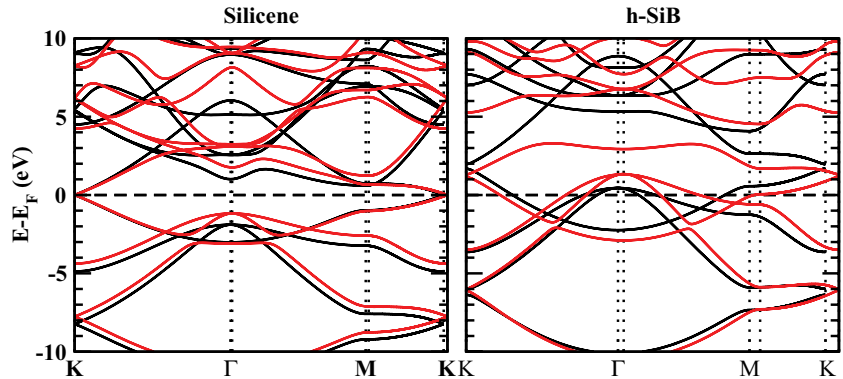

FIG. 10. (Color online) Band structure of planar and buckled sheets of silicene and h-SiB. Red (black) lines corresponds to the buckled (planar) structures. The more stable (buckled) $\beta$-silicene sheet has a $\Delta$ of $0.51 \AA$, while the unstable buckled h-SiB has a $\Delta$ of $0.70 \AA$.

results, ${ }^{62}$ while the more accurate $G W$ approximation gives a band gap of $6.47 \mathrm{eV}$. The three hexagonal sheets have typical band structures of honeycomb sheets, however, the widths and positions of the bands vary, mainly due to the number of core and valence electrons of the constituent atoms. In contrast to graphene, the $\mathrm{h}-\mathrm{SiB}$ sheet has a narrow pseudo band gap about $0.40 \mathrm{eV}$ at the $K$ point between the conduction $\left(\pi^{*}\right)$ band and valence $(\pi)$ band. On the other hand, the $\pi$ band and the two highest occupied $\sigma$ bands are only partly filled, resulting in a metallic behavior of the $\mathrm{h}-\mathrm{SiB}$ sheet.

The DOS and partial/projected DOS (PDOS) of h-BN differ more from graphene than $\mathrm{h}-\mathrm{SiB}$ does (except for the Fermi energy position), which is also seen in the band structures. Particularly in the $\pi$ bands of h-BN, the $2 p_{z}$ orbital of nitrogen contributes much more to the first (valence) $\pi$ band, while the $2 p_{z}$ orbital of boron contributes more to the second (conduction) $\pi$ band, which explains the more localized charge density in nitrogen of h-BN. In the case of h-SiB, the $3 p_{z}$ orbital of silicon and the $2 p_{z}$ orbital of boron give similar contributions to the $\pi$ bands. The first (lowest) and more narrow $\sigma$ bands of h-BN, also have a higher representation of the $2 p_{ \pm 1}$ orbitals (of boron) than graphene and h-SiB. As proposed previously, the planar structure of $\mathrm{h}-\mathrm{SiB}$ indicates an $s p^{2}$ hybridization with the overlapping $2 p_{z}$ orbitals of boron and with $3 p_{z}$ orbitals of silicon with charge transfer from $3 p_{z}$ to $2 p_{z}$ (see Fig. 8).

In the case of pure silicon, the $s p^{2}$-hybridized planar $\alpha$-silicene is predicted to be semi-metallic-like graphene ${ }^{18,22}$ in contrast to the semiconducting $s p^{3}$-hybridized bulk silicon. If bulk silicon is viewed as stacked buckled silicene sheets, the buckling parameter is about $0.80 \AA$. Therefore, the
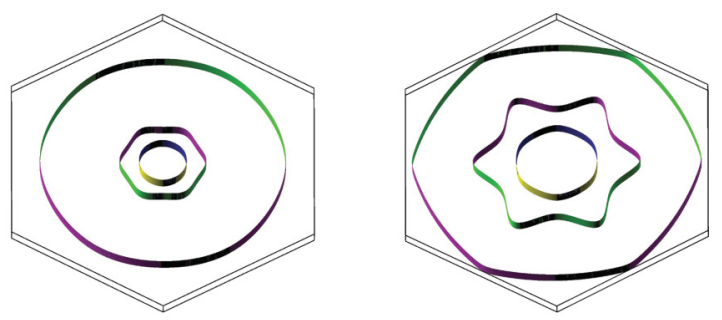

FIG. 11. (Color online) The 2D Fermi surfaces (in slightly tilted $3 \mathrm{D}$ plots) of the planar stable h-SiB sheet (left) and the unstable buckled $(\Delta=0.70 \AA) \mathrm{h}-\mathrm{SiB}$ sheet (right).
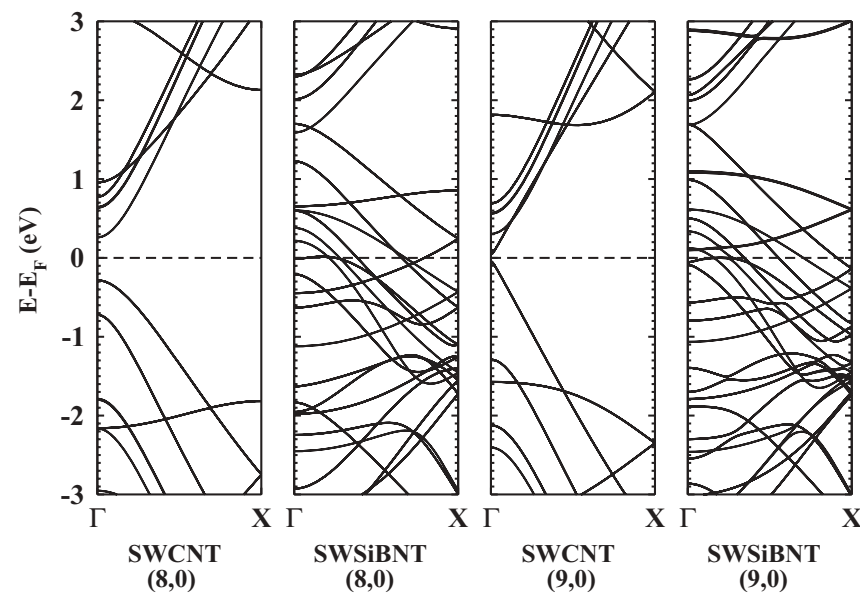

FIG. 12. Band structures of the zigzag $(8,0)$ and $(9,0)$ SWCNTs and the corresponding SWSiBNTs. The Fermi energy is indicated by a dashed line.

less buckled $\beta$-silicene indicates a hybridization between $s p^{2}$ and $s p^{3}$ with either a semimetallic or semiconducting behavior, depending on the degree of buckling. In some studies reviewed by Kara et $a l^{22} \beta$-silicene is predicted to remain semimetallic (including this study; see Fig. 10), while Behera and Mukhopadhyay ${ }^{63}$ report a small direct band gap of about $25 \mathrm{meV}$ at the $K$ point.

To investigate the sensitivity of the metallic behavior with respect to buckling of the $\mathrm{h}-\mathrm{SiB}$ sheet, the band structure of the unstable buckled h-SiB sheet with a $\Delta$ of $0.70 \AA$ has been calculated (displayed in Fig. 10). The rather large buckling for a honeycomb sheet with bond lengths of $1.99 \AA$ shifts the bands slightly around the Fermi energy, but does not open a band gap. The Fermi surfaces of both the planar and buckled h-SiB are displayed in Fig. 11. The outermost contour is assigned to the $\pi$ band, whereas the two inner contours are assigned to the $\sigma$ bands. Note that the effect of buckling leads to a significant change of the inner contours of the Fermi surface, although it preserves the metallic character of the system.

The curvature of nanotubes also affects the $s p^{2}$ hybridization found in the corresponding sheets. Fagan et al. ${ }^{59}$ have shown that the less stable $\alpha$-silicene nanotubes, which are structural analogs of carbon nanotubes, have electronic properties very similar to the equivalent carbon nanotubes, i.e., depending on their chiralities, they may have metallic or semiconducting behavior. On the other hand, the more stable buckled ( $\beta$-silicene) nanotubes, have more semiconducting chiralities (both armchair and zigzag). ${ }^{15}$

The corresponding nanotubes, SWSiBNTs, may also form metallic systems, once the curvature does not introduce a band gap. As a consequence of the partly filled bands in $\mathrm{h}-\mathrm{SiB}$, also the zigzag tubes will be metallic. The zone-folding approximation, ${ }^{64}$ used for understanding the band structure of carbon nanotubes, predicts the SWSiBNTs to be metallic independently of their chirality. This is confirmed by the calculated band structures for all the studied SWSiBNTs. In Fig. 12 we show two examples for $(8,0)$ and $(9,0)$ SWCNTs. As noticed, the $(9,0)$ SWCNT, which is metallic within the zone-folding approximation, has a band gap of $0.09 \mathrm{eV}$ 
(in agreement with Ref. 65), while the SWSiBNTs form truly metallic systems.

\section{CONCLUSIONS}

The present study has examined the structural and electronic properties of graphenelike structures made of silicon and boron elements, as well as some single-walled nanotubes. Based on first-principles structure optimization and stability calculations, we have proposed a planar silicon boron honeycomb crystal, i.e., h-SiB, which exhibits structural stability near the values obtained for silicene. The calculated cohesive energy of the h-SiB system is slightly lower than bulk silicon but larger than silicene. More deeply studied in this paper, $\mathrm{h}-\mathrm{SiB}$ appears to be an interesting prototype of a 2D metallic system. In particular, this sheet exhibits a $\pi$ electron density at the $\Gamma$ point similar to that observed in graphene, but with a difference of energies between orbitals localized on $\mathrm{Si}$ and B. Our electronic structure calculations predict a metallic behavior due to a partially filled valence band and a region of high density of states around the Fermi energy.

The h-SiB sheets are not able to stack into 3D graphitelike structures, but give rise to wurtzitelike structures. However, we believe that the relatively weaker $s p^{2}$ hybridization of the silicon boron sheets can remain while embedded in other layered structures, as observed for silicene. Folded into tubes (SWSiBNTs), the h-SiB sheet has a strain energy four to five times less than graphene/SWCNTs, nevertheless it is sufficient for giving the smaller tubes a cylindrical shape. Also, AIMD simulations have predicted that these nanotubes exhibit a good thermal stability at high temperatures. In contrast to the SWCNTs, for which the metallicity depends on the chirality, both armchair and zigzag SWSiBNTs are predicted to be metallic.

Results from this study further suggest that $\mathrm{h}-\mathrm{SiB}$ could be explored as a candidate material for applications in lowdimensional electronic devices. We expect that 2D systems such as h-SiB sheets can be stabilized by transverse shortrange atomic displacements. ${ }^{66}$ Furthermore, studies ${ }^{67}$ on the structural stability and variety of single boron layers have demonstrated that such systems are stable and metallic. In this sense, recent experiments of chemical vapor deposition of $\alpha$-B layers on silicon ${ }^{68}$ could open up interesting perspectives in obtaining nanometer-thick ordered low-dimensional $\mathrm{SiB}$ structures. Our results also open up new possibilities for the realization of metallic graphenelike systems in electronic transport.

\section{ACKNOWLEDGMENTS}

Financial support from the National Council of Scientific and Technological Development $(\mathrm{CNPq})$ and computational facilities at the National High-Performance Computing Center in São Paulo (CENAPAD-SP) are gratefully acknowledged. We also thank R. Veiga and D. Tománek for sharing the TubeASP utility. *hansson@linux.com

†fbmota@ufba.br

${ }^{\ddagger}$ rivelino@ufba.br

${ }^{1}$ R. E. Peierls, Ann. Inst. Henri Poincare 5, 177 (1935).

${ }^{2}$ L. D. Landau, Phys. Z. Sowjetunion 11, 26 (1937).

${ }^{3}$ N. D. Mermin, Phys. Rev. 176, 250 (1968).

${ }^{4}$ A. K. Geim and K. S. Novoselov, Nat. Mater. 6, 183 (2007).

${ }^{5}$ P. W. Anderson, Phys. Rev. 109, 1492 (1958).

${ }^{6}$ P. R. Wallace, Phys. Rev. 71, 622 (1947).

${ }^{7}$ J. W. McClure, Phys. Rev. 104, 666 (1956).

${ }^{8}$ J. C. Slonczewski and P. R. Weiss, Phys. Rev. 109, 272 (1958).

${ }^{9}$ A. H. Castro Neto, F. Guinea, N. M. R. Peres, K. S. Novoselov, and A. K. Geim, Rev. Mod. Phys. 81, 109 (2009).

${ }^{10}$ K. S. Novoselov, A. K. Geim, S. V. Morozov, D. Jiang, Y. Zhang, S. V. Dubonos, I. V. Grigorieva, and A. A. Firsov, Science 306, 666 (2004).

${ }^{11}$ K. S. Novoselov, A. K. Geim, S. V. Morozov, D. Jiang, M. I. Katsnelson, I. V. Grigorieva, S. V. Dubonos, and A. A. Firsov, Nature (London) 438, 197 (2005).

${ }^{12}$ T. Suzuki and Y. Yokomizo, Physica E 42, 2820 (2010).

${ }^{13}$ E. Bekaroglu, M. Topsakal, S. Cahangirov, and S. Ciraci, Phys. Rev. B 81, 075433 (2010).

${ }^{14}$ L.-C. Yin and R. Saito, J. Phys. Soc. Jpn. 80, 114707 (2011).

${ }^{15}$ X. Yang and J. Ni, Phys. Rev. B 72, 195426 (2005).

${ }^{16}$ C. Léandri, H. Oughaddou, B. Aufray, J. Gay, G. L. Lay, A. Ranguis, and Y. Garreau, Surf. Sci. 601, 262 (2007).
${ }^{17}$ A. Kara, C. Léandri, M. Dávila, P. De Padova, B. Ealet, H. Oughaddou, B. Aufray, and G. Le Lay, J. Supercond. Novel Magn. 22, 259 (2009).

${ }^{18}$ S. Lebègue and O. Eriksson, Phys. Rev. B 79, 115409 (2009).

${ }^{19}$ S. Cahangirov, M. Topsakal, E. Aktürk, H. Şahin, and S. Ciraci, Phys. Rev. Lett. 102, 236804 (2009).

${ }^{20}$ B. Aufray, A. Kara, S. Vizzini, H. Oughaddou, C. Leandri, B. Ealet, and G. L. Lay, Appl. Phys. Lett. 96, 183102 (2010).

${ }^{21}$ M. Houssa, G. Pourtois, V. V. Afanas'ev, and A. Stesmans, Appl. Phys. Lett. 97, 112106 (2010).

${ }^{22}$ A. Kara, H. Enriquez, A. P. Seitsonen, L. L. Y. Voon, S. Vizzini, B. Aufray, and H. Oughaddou, Surf. Sci. Rep. 67, 1 (2012).

${ }^{23}$ D. Golberg, Y. Bando, Y. Huang, T. Terao, M. Mitome, C. Tang, and C. Zhi, ACS Nano 4, 2979 (2010).

${ }^{24}$ K. S. Novoselov, D. Jiang, F. Schedin, T. J. Booth, V. V. Khotkevich, S. V. Morozov, and A. K. Geim, Proc. Natl. Acad. Sci. USA 102, 10451 (2005).

${ }^{25}$ H. Şahin, S. Cahangirov, M. Topsakal, E. Bekaroglu, E. Akturk, R. T. Senger, and S. Ciraci, Phys. Rev. B 80, 155453 (2009).

${ }^{26}$ S. Limpijumnong and W. R. L. Lambrecht, Phys. Rev. B 63, 104103 (2001).

${ }^{27}$ F. Claeyssens, C. L. Freeman, N. L. Allan, Y. Sun, M. N. R. Ashfold, and J. H. Harding, J. Mater. Chem. 15, 139 (2005).

${ }^{28}$ C. L. Freeman, F. Claeyssens, N. L. Allan, and J. H. Harding, Phys. Rev. Lett. 96, 066102 (2006).

${ }^{29}$ C. Tusche, H. L. Meyerheim, and J. Kirschner, Phys. Rev. Lett. 99, 026102 (2007). 
${ }^{30}$ Y. Duan, L. Qin, G. Tang, and L. Shi, Eur. Phys. J. B 66, 201 (2008).

${ }^{31}$ B. J. Morgan, Phys. Rev. B 80, 174105 (2009).

${ }^{32}$ A. Wander, F. Schedin, P. Steadman, A. Norris, R. McGrath, T. S. Turner, G. Thornton, and N. M. Harrison, Phys. Rev. Lett. 86, 3811 (2001).

${ }^{33}$ J. M. Carlsson, Comput. Mater. Sci. 22, 24 (2001).

${ }^{34}$ R. J. Cava, H. Takagi, B. Batlogg, H. W. Zandbergen, J. J. Krajewski, W. F. Peck, R. B. van Dover, R. J. Felder, T. Siegrist, K. Mizuhashi, J. O. Lee, H. Eisaki, S. A. Carter, and S. Uchida, Nature (London) 367, 146 (1994).

${ }^{35}$ J. Nagamatsu, N. Nakagawa, T. Muranaka, Y. Zenitani, and J. Akimitsu, Nature (London) 410, 63 (2001).

${ }^{36}$ E. A. Ekimov, V. A. Sidorov, E. D. Bauer, N. N. Mel'nik, N. J. Curro, J. D. Thompson, and S. M. Stishov, Nature (London) 428, 542 (2004).

${ }^{37}$ M. Kriener, T. Muranaka, Z.-A. Ren, J. Kato, J. Akimitsu, and Y. Maeno, J. Phys.: Conf. Ser. 150, 052130 (2009).

${ }^{38}$ E. Bustarret, C. Marcenat, P. Achatz, J. Kačmarčik, F. Lévy, A. Huxley, L. Ortéga, E. Bourgeois, X. Blase, D. Débarre, and J. Boulmer, Nature (London) 444, 465 (2006).

${ }^{39}$ T. L. Aselage and D. R. Tallant, Phys. Rev. B 57, 2675 (1998).

${ }^{40}$ E. Colton, J. Am. Chem. Soc. 82, 1002 (1960).

${ }^{41}$ E. Dominguez, E. Lora-Tamayo, B. Blanchard, and J. Bellanato, J. Electrochem. Soc. 125, 1521 (1978).

${ }^{42}$ M. Vlasse, G. A. Slack, M. Garbauskas, J. S. Kasper, and J. C. Viala, J. Solid State Chem. 63, 31 (1986).

${ }^{43}$ J. M. Soler, E. Artacho, J. D. Gale, A. García, J. Junquera, P. Ordejón, and D. Sánchez-Portal, J. Phys.: Condens. Matter 14, 2745 (2002).

${ }^{44}$ J. P. Perdew, K. Burke, and M. Ernzerhof, Phys. Rev. Lett. 77, 3865 (1996).

${ }^{45}$ S. Dutta, A. K. Manna, and S. K. Pati, Phys. Rev. Lett. 102, 096601 (2009).

${ }^{46}$ N. Troullier and J. L. Martins, Phys. Rev. B 43, 1993 (1991).

${ }^{47}$ N. W. Ashcroft and D. N. Mermin, Solid State Physics, 1st ed. (Thomson Learning, Toronto, 1976).

${ }^{48}$ T. He, M. Zhao, W. Li, C. Song, X. Lin, X. Liu, Y. Xia, and L. Mei, J. Phys. Chem. C 111, 16840 (2007).
${ }^{49}$ J. Kunstmann, A. Quandt, and I. Boustani, Nanotechnology 18, 155703 (2007).

${ }^{50}$ W. Sheng-Jie, Z. Chun-Lai, and W. Zhi-Guo, Chin. Phys. Lett. 27, 106101 (2010).

${ }^{51}$ CRC Handbook of Chemistry and Physics, internet version 2006 ed., edited by D. R. Lide (Taylor and Francis, Boca Raton, FL, 2006).

${ }^{52}$ V. L. Solozhenko, G. Will, and F. Elf, Solid State Commun. 96, 1 (1995).

${ }^{53}$ A. R. Oganov, J. Chen, C. Gatti, Y. Ma, Y. Ma, C. W. Glass, Z. Liu, T. Yu, O. O. Kurakevych, and V. L. Solozhenko, Nature (London) 457, 863 (2009).

${ }^{54}$ B. Farid and R. W. Godby, Phys. Rev. B 43, 14248 (1991).

${ }^{55}$ P. V. C. Medeiros, F. de Brito Mota, A. J. S. Mascarenhas, and C. M. C. de Castilho, Nanotechnology 21, 115701 (2010).

${ }^{56}$ R. W. Lynch and H. G. Drickamer, J. Chem. Phys. 44, 181 (1966).

${ }^{57}$ P. Vogt, P. De Padova, C. Quaresima, J. Avila, E. Frantzeskakis, M. C. Asensio, A. Resta, B. Ealet, and G. Le Lay, Phys. Rev. Lett. 108, 155501 (2012).

${ }^{58}$ Y. Ding and J. Ni, Appl. Phys. Lett. 95, 083115 (2009).

${ }^{59}$ S. B. Fagan, R. J. Baierle, R. Mota, A. J. R. da Silva, and A. Fazzio, Phys. Rev. B 61, 9994 (2000).

${ }^{60}$ K. Abersfelder, A. J. P. White, H. S. Rzepa, and D. Scheschkewitz, Science 327, 564 (2010).

${ }^{61}$ B. Arnaud, S. Lebègue, P. Rabiller, and M. Alouani, Phys. Rev. Lett. 96, 026402 (2006).

${ }^{62}$ L. Song, L. Ci, H. Lu, P. B. Sorokin, C. Jin, J. Ni, A. G. Kvashnin, D. G. Kvashnin, J. Lou, B. I. Yakobson, and P. M. Ajayan, Nano Lett. 10, 3209 (2010).

${ }^{63} \mathrm{H}$. Behera and G. Mukhopadhyay, arXiv:1201.1164v1.

${ }^{64}$ R. Saito, G. Dresselhaus, and M. S. Dresselhaus, Physical Properties of Carbon Nanotubes (Imperial College Press, London, 1998).

${ }^{65}$ T. Miyake and S. Saito, Phys. Rev. B 72, 073404 (2005).

${ }^{66}$ A. O'Hare, F. V. Kusmartsev, and K. I. Kugel, Nano Lett. 12, 1045 (2012).

${ }^{67}$ E. S. Penev, S. Bhowmick, A. Sadrzadeh, and B. I. Yakobson, Nano Lett. 12, 2441 (2012).

${ }^{68}$ F. Sarubbi, T. Scholtes, and L. Nanver, J. Electr. Mat. 39, 162 (2010). 